\title{
DETERMINATION OF PROGNOSIS OF SNAKE BITE CASES BY NEUTROPHIL-LYMPHOCYTE RATIO AND PLATELET-LYMPHOCYTE RATIO.
}

\section{Dr.Ravi Allichandi}

\section{Dr. Manjunath Kurahatti*} ABSTRACT

\author{
Assistant Prof, Dept of General Medicine,SNMC, Bagalkot, Karnataka.
}

\author{
Junior Resident, Dept of General Medicine,SNMC, Bagalkot, Karnataka. \\ ${ }^{*}$ Corresponding Author
}

ABSTRACT OBJECTIVES: Neutrophil-lymphocyte ratio (NLR) and platelet-lymphocyte ratio (PLR) have recently been suggested as un result in serious complications. Our country is a snakebite-endemic area. The parameters for the prognosis of snakebites are very valuable due to the inadequacy of acute inpatient services, as well as the excessively intense traffic at emergency rooms (ERs); therefore, we aimed to detect the importance of the neutrophil-lymphocyte ratio (NLR) and the platelet-lymphocyte ratio (PLR) as parameters in the prognosis for snakebites. METHODS: In this study, patients who presented at the ER within 24 hours after a snakebite were retrospectively analyzed. The sex, age, exposure duration, body part affected, hospitalization period, complications, mortality rate, and blood parameters of ER patients were assessed. RESULTS: 51 healthy individuals who were suitable in terms of sex and age were included in the control group. It was detected that the average NLR ( $p=0.001)$ and PLR $(p=0.005)$ values were statistically significantly higher in the patient group than in the control group. On comparing the patients discharged from the ER with those who remained hospitalized, the NLR $(p=0.002)$ and PLR $(p=0.006)$ values were significantly higher in the patients who remained hospitalized. The NLR $(p=0.004)$ and the PLR $(p=0.003)$ values in the patients who developed complications were significantly higher than those in patients who did not develop complications. CONCLUSION: High levels of on admission NLR and PLR were associated with worse clinical profile and poorer outcome. The hospitalization period, complication developments, and poor prognosis in snakebites may be predicted using the NLR and PLR values, which are easily available and inexpensive blood parameters.

\section{KEYWORDS : Neutrophil-lymphocyte Ratio (nir), Platelet-lymphocyte Ratio (plr), Snakebites}

\section{INTRODUCTION}

Snake bite envenomation is a common acute life- threatening medical emergency. More than 200,000 snake bites are reported in the country and an estimated 35000 to 50000 people die each year. Majority of snake bite deaths go unreported as many victims go to traditional healers and many deaths occur before reaching hospital.(1)

Although the total number of snake species present throughout the world is not precisely known, it is presumed to be between 2,500 and 3,000. Only $1 / 3$ rd of the snake species is venomous, which can be classified as dangerous to mankind. Only $8 \%$ of the snake species is dangerous to mankind (2). There are about 216 species of snakes identifiable in india, of which 52 are poisonous. The major families of poisonous snakes in india are Elapidae which includes Common Cobra and common Krait, Viperidae. Snakebite is a significant mortality and morbidity cause, which occurs particularly in the summer months.(1)

Venom secretion in all venomous snakes appears to vary in seasons. In warmer months, its output is more than in the cold season.(1)

The snake venom comprises of a complicated structure, comprising an aggregation of various toxic proteins and enzymes. The snake venom has cardiotoxic, neurotoxic, miotoxic, nephrotoxic, and hematotoxic characteristics, and local and systemic symptoms are observed in patients based on the intensity of the toxin. $(3,4)$

Many studies have reported that many complications such as necrosis, amputation, acute renal failure, compartment syndrome, and mortality were encountered arising out of snakebites. $(5,6,7)$

In the hospitals where snakebite cases are frequently encountered and the inpatient bed availability is limited, follow- up is challenging and the patients return with further complications after being discharged from the hospital. Therefore, determining the prognosis is of vital importance, as the patient applies to the hospital.

Treatment for poisonous snakebite is divided into supportive care and anti-venom administration. However, there is no standardized guideline on anti-venom administration and depending solely on severity of symptoms at presentation for clinical assessment is not reliable. It is very difficult to predict the expansion of localized and systematic symptoms. In some cases, generalized symptoms develop in the absence of localized symptoms. It is believed that when the initial dose is not adequate and/or delayed, it might worsen the morbidity and mortality rates. (8)

Bedside investigations would be ideal, but international normalized ratio (INR) and whole blood clotting tests have been shown to be unreliable in many settings especially in critically ill patients with multi-organ failure as a result of snake bite. $(9,10)$. Thus, it is important that the health care providers should be able to identify patients with snakebites at high risk of fatal complications early enough so as to be able to ensure optimum and timely management.

In recent years, various studies have been conducted on the capability of prognosis and mortality predictions for many diseases with NLR and PLR, which are easily available and inexpensive blood parameters.[1 1,12,13]

Reflecting the neutrophil level for the acute state in inflammation, and the lymphopenia developing after acute physiological stress, NLR was used with other inflammatory markers in the studies, thus recognized to be an efficient marker for inflammatory state.[13]

Recently, it has been reported that PLR is a potential marker of inflammation and is an independent predictor of mortality in cardiac and various oncological diseases. [14] 
In this study, we aimed to analyze the role of NLR and PLR values measured during admission for determining poor prognosis in the early period for patients presenting to ER with snakebite.

\section{METHODOLOGY:}

Study design: Retrospective study.

Source of study population: Snake Bite Cases admitted at SNMC HSK hospital.

\section{Inclusion criteria:}

Snake Bite cases admitted between January 2018 to September 2020 were retrospectively analysed. The patient information was accessed through the hospital's records and automation system.

\section{Exclusion criteria:}

The patients who were diagnosed with idiopathic thrombocytopenic purpura, thrombotic thrombocytopenic purpura, and essential thrombocytosis; those who had a history of medication use leading to the disorders of platelets and neutrophils structure and function; and those with missing data were excluded.

\section{Sample size:}

Title of the Article - Importance of Neutrophil-Lymphocyte Ratio and Platelet-Lymphocyte Ratio for Determining the Prognosis and Hospitalization Period in Patients with Snckebites

\section{Author - Nazli Gormeli Kurt, Murat Orak, Mehmet Ustundag}

Journal- Eurasian Journal of Medical Investigation (EJMI)

Date - 2018

By this article,

Sample size of 51 in each group ( cases and controls) was obtained from OpenEpi, Version 2, open source calculatorSSMean.

\section{Data collection:}

- The following characteristics and parameters were analyzed for the 51 patients: patients' sex, age, profession, vital findings, duration of exposure, bitten body part, hospitalization period, complications developed, mortality rate, including the blood parameters as applying to the hospital.

- Fifty one healthy individuals who were suitable in terms of sex and age were included in the control group.

- The cases were split into the following paired groups: patients and controls; those who developed complications and those who did not; those who remained hospitalized and those who were discharged from the hospital; and lower extremity and upper extremity snakebites. The NLR and PLR values of these groups were compared.

Investigations:

CBC, Urea, Creatinine.

Plan for statistical analysis:

In univariate statistical analyses, chi-square test was applied for categorical variables, whereas Student-t test was applied for continuous variables. Numeric variables were stated as mean \pm SD. $P<0.05$ was considered as statistically significant.

Pearson correlation analysis was performed for the measurement of linear relationship level between two continuous variables.

\section{RESULTS :}

51 patients presented with snakebite to the ER of SNMC and HSK Hospital, Bagalkot,were selected, between January 2018 to September 2020. In the study, 66.66\% (34) of the patients were male and $33.33 \%$ (17) were female. We calculated the age average of the patients as $52.94 \%$ (27) of the control group was male, while $47.05 \%$ (24) of the group was female; the age average was $40.36 \pm 15.48$ years. There was no statistically significant difference regarding age and sex between the control and patient groups.

$54 \%$ of the patients $(n=28)$ were bitten in the lower extremity and $45 \%(n=23)$ were bitten in the upper extremity. Also, 37.3\% $(\mathrm{n}=19)$ of the patients were discharged after treatment and monitored in ER, while $62.7 \%(n=32)$ of them remained hospitalized. The average hospitalization period of the patients who remained hospitalized was $7.86 \pm 5.43$.

In addition, $15.7 \%(n=8)$ of the 51 patients developed complications while being monitored and 3 of them developed compartment syndrome, while 1 of these complications were finger amputations, 2 were coagulopathy , 1 with acute renal failure and $l$ with sepsis and death.

Table 1. Comparison of patient and control groups for their NLR and PLR values

\begin{tabular}{|l|l|l|l|}
\hline & $\begin{array}{l}\text { Patients } \\
(\mathrm{n}=51)\end{array}$ & $\begin{array}{l}\text { Controls } \\
(\mathrm{n}=51)\end{array}$ & $\begin{array}{l}\mathrm{p} \\
\text { value }\end{array}$ \\
\hline NLR (mean +/- SD) & $9.06+/-5.27$ & $3.33+/-2.53$ & 0.001 \\
\hline PLR ( mean +/- SD) & $179.96+/-123.71$ & $121.12+/-84.07$ & 0.005 \\
\hline
\end{tabular}

We analyzed the NLR and PLR values of the patient and control groups as applying to the hospital, involved in our study. We detected that the average NLR $(p=0.001)$ and PLR $(p=0.005)$ values were statistically significantly higher in the patient group than in the control group (Table 1).

Table 2. Relationship between the NLR and PLR values and treatment process (discharge, hospitalization), development of complication, and bitten body part

\begin{tabular}{|l|l|l|l|l|l|l|}
\hline & & (n) & NLR (mean+/-SD) & p value & PLR (mean +/- SD) & p value \\
\hline Treatment process & Discharge & 19 & $6.07+/-3.13$ & 0.002 & $117.05+/-45.49$ & 0.006 \\
\hline & hospitalisation & 32 & $10.6+/-5.52$ & & $211.96+/-139.13$ & \\
\hline Complication & Developing & 8 & $13.6+/-4.38$ & 0.004 & $300.16+/-147.93$ & 0.003 \\
\hline & Not developing & 43 & $8.1+/-4.88$ & & $164.54+/-106.09$ & \\
\hline Bitten body part & Lower extremity & 28 & $8.89+/-5.54$ & 0.82 & $190.87+/-126.33$ & 0.49 \\
\hline & Upper extremity & 23 & $9.23+/-5.03$ & & $166.88+/-121.91$ & \\
\hline
\end{tabular}

Comparing the patients who were discharged from ER and those who remained hospitalized, the NLR value $(p=0.002)$ and PLR values were significantly higher in the patients who remained hospitalized ( $\mathrm{p}=0.006$ ) (Table 2$)$.

The NLR $(p=0.004)$ and PLR $(p=0.003)$ values in the patients who developed complications were significantly higher than those in the patients who did not develop complications.

Being bitten on the lower or upper extremity by the snake did not constitute any significant change on the NLR $(p=0.82)$ and $\operatorname{PLR}(\mathrm{p}=0.49)$ values. 
Fig.l : Relation between hospitalization period and NLR value

\section{NLR}

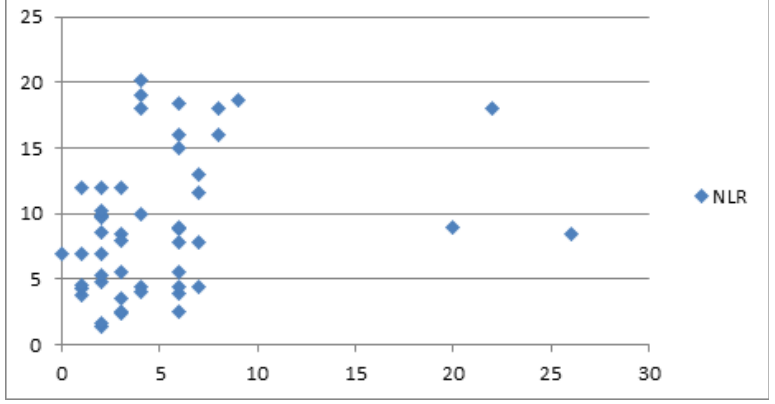

$\mathrm{X}$ axis $=$ Hospitalisation, $\mathrm{Y}$ axis $=$ NLR, Pearson correlation coefficient $(r)=0.28$

Fig 2 : : Relation between hospitalization period and PLR value

\section{PLR}

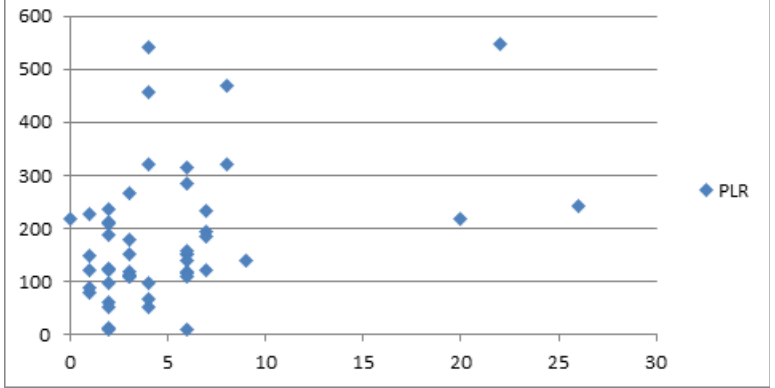

$\mathrm{X}$ axis $=$ Hospitalisation, $\mathrm{Y}$ axis $=\mathrm{PLR}$, Pearson correlation coefficient $(r)=0.384$

We analyzed the relation between the hospitalization period and blood parameters of the patients and detected $a$ positive correlation between the NLR

$(\mathrm{p}=0.000, \mathrm{r}=0.281)$ and PLR $(\mathrm{p}=0.000, \mathrm{r}=0.384)$ values and hospitalization period (Fig. 1, Fig. 2).

\section{DISCUSSION}

- Snakebite is one of the most common causes of admission to ER within our region, particularly in the summer months.

- The Viperidae family of snakes is frequently seen in this region due its geographical position, and snakebites are considered as a serious public health problem, since the temperature is above seasonal normal and the people mainly work in rural areas in the summer months.

- Snake poisoning course may be fulminant and lethal thus recognition of prompt parameter could reduce the mortality.(9)

- Preliminary evidence has suggested that, leukocytes and their subtypes (granulocytes, monocytes and lymphocytes) counts can be used as a marker of inflammation in many diseases. (15)

- When the differential count of leukocytes is evaluated during the acute inflammatory response to oxidative stress, it is characterized by an increase in neutrophil and monocyte counts and a decline in lymphocyte counts. $(16,17)$

- In this study there was significant increase of TLC and neutrophil counts and a significant decrease of lymphocyte count in the moderate and severe groups of snake poisoned patients in comparison to the mild and control groups. This is similar to studies by Moreira et al.
(2009); Zornetta et al. (2012); Elbey et al. (2015) $(18,19,20)$ who reported significant leukocytosis, neutrophilia and lymphocytopenia in patients with snake poisoning.

- The inflammatory properties of snake toxicity are attributed to its venom composition (19).

- In circulatory system, snake venoms not only affect vessel wall, platelet function, clot formation, and clot dissolution, but also target blood cells. Cells of the innate immunity, the white blood cells, are highly sensitive and take part in the venom-induced inflammatory and oxidant response. (21) (Sharma et al., 2015).

- A study by Chacon et al. (2015) (22) reported common inflammatory events following viperidae snake bites envenomation. Significant release of pro-inflammatory mediators as; Interleukin (IL)-6, IL-10, tumor necrosis factor alpha (TNF ) together with surge of reactive oxygen species (ROS) indicating obvious inflammatory and oxidant stress following snake envenoming was noticed by Acikalin and Gokel (2011) (23); Stone et al. (2013) (24) ; Sharma et al. (2015) (21).

- In a study by Al Durihim et al. (2010) (25) leukocytosis was an encountered finding in the complete blood count of patients admitted to hospital with snakebite.

- In another study by Isbister et al. (2016) (26) leukocytosis was evident even before detection of significant coagulopathy and prolonged PTT following snake poisoning. A correlation between snake evenomations and sterile inflammatory syndrome and innate immune response that might contribute to the local and systemic inflammatory events with induction of neutrophil activation is suggested by Zornetta et al. (2012) (19).

- Elevated neutrophil count starts from the first hour due to demargination of neutrophils from the endothelium, delay of neutrophil apoptosis, and effect of growth factors on stem cells (27). Whereas, lymphocytopenia occurs in response to physiological stress-induced margination to the reticulo-endothelial system, redistribution and accelerated apoptosis .(28)

- The increase of neutrophil and the decrease of lymphocyte counts explain the on admission pretreatment high levels of neutrophil-lymphocyte ratio (NLR) and Platelet Lymphocyte ratio (PLR) in this study among the moderate and severe groups of snake envenomated patients. This is in accordance with a study by Elbey et al. (2015). (20)

- NLR is a recently defined novel inflammatory marker which has emerged as a readily available, valuable and reliable method for follow-up of a number of local and systemic inflammatory diseases. (29) Its prognostic value was proved in non-poisoning settings as; cardiovascular and pulmonary diseases and related co-morbidities detection, as well as in diverse immuneinflammatory diseases (30). NLR was also shown to be a predictor of survival and response to treatment in various types of malignancies.

- In the current study, there was significant correlation between NLR and Platelet-lymphocyte ratio (PLR), hospital stay time and intensive care unit (ICU) stay time. NLR was higher in the severe and complicated cases. This association of on admission pre-treatment high NLR with worse outcome indicates the value of NLR as a prognostic factor of snake poisoning. Similarly, Elbey et al. (2015) (20)found that NLR was significantly increased in patients who developed complications and needed a longer hospitalization.

- Salciccioli et al. (2015) (31) stated that mortality was higher in patients with increased NLR. Although complications occurred in some cases of snake bites in the severe group, only one case died of multiple organ failure (MOF) in this study. 
- Elevated NLR is believed to be associated with increased in-hospital and post-hospital mortality (32).

- Akilli et al. (2014) (27) stated that NLR provide an accurate guidance for follow up and therapeutic intervention and can also enlighten the physician to the development of MOF and sepsis, particularly within a few hours after admission in critically ill patients.

- Another study on 90 ICU patients stated that NLR was more reliable parameter for reflecting the intensity of stress, evaluating and monitoring systemic inflammatory response and predicting short and long mortality (33).

- In poisoning settings associated with oxidative stress, NLR was found to be useful for estimating prognosis and follow up in pesticide and herbicide poisoned patients $(8,17)$. Elevated NLR has been shown to be associated with increased TNF and various interleukins (IL-6, IL-7, IL-8, IL-12, IL-17).These markers (previously mentioned to be involved in snake venom toxicity) are known to be associated with poor outcome in critically ill patients. However, measuring these biomarkers is expensive and is not routinely performed. In contrast, NLR is a simple index derived from routine blood tests which might provide equal and valuable information. Because it is a ratio, NLR is relatively more stable than individual leukocytic parameters that are easily altered by many simple conditions (eg. dehydration, over hydration, diluted blood specimens, and in vitro blood specimen handling).

- Besides NLR, platelets are found to be associated with disease activity in patients with inflammatory disease. They play a significant role in inflammation and immunity, besides being major elements of hemostasis (14). Plateletlymphocyte ratio (PLR) is a new biomarker indicating presence and severity of inflammation. Like NLR, PLR in peripheral blood is easy, readily available systemic inflammatory response.

- Elevated PLR levels are frequently observed in nonpoisoning conditions as; in various malignancies, cardiac diseases and immune-inflammatory diseases (34) and in poisoning conditions with oxidative stress as in pesticide poisoning where it is associated with bad prognosis and high mortality rate but with lesser efficacy than NLR (17).

- Local and systemic symptoms are observed in patients depending on the density of the snake venom. The local findings are edema, hematoma, and gangrenous lesions, while the systemic findings are pyrexia, nausea, vomiting, circulatory failure, mild hepatitis, delirium, convulsion, and coma. Mortality may occur due to secondary infections, disseminated intravascular coagulopathy, neurotoxicity, acute renal failure, intracranial hemorrhage. Besides, progressing anemia, leukocytosis, thrombocytopenia, hyperfibrinogenemia, failure in coagulation tests, proteinuria, and azotemia have also been reported.[35]

- Snake venom contains phospholipase, acetylcholinesterase, hyaluronidase, collagenase, RNA and DNA, leukotrienes, antibactericidin, neurotoxins, procoagulant, anticoagulant, cardiotoxin, hemotoxin, and certain electrolytes.It also causes secretion of quinine, histamine, and serotonin via cytolytic enzymes in the bitten area. Apart from the aforementioned, the snake venom also contains proteolytic and hemolytic factors, amino-acid oxidants, as well as other enzymes. (36) These enzymes, contained within the venom, may generate antiinflammatory response, apart from tissue and cell damage.

- We analyzed the relationship between the NLR and PLR values, as anti-inflammatory parameters, and poor prognosis, development of complication, and hospitalization period in patients who presented to the hospital with snakebites.

- We confirmed that the NLR and PLR values may be significant in poor prognosis.
- Elbey et al.(20) confirmed a significant relation between NLR value and poor prognosis in his studies on snakebites. Apart from obtaining a similar result in our study, we also confirmed that PLR value comprises a predictive value concerning poor prognosis, development of complication, and hospitalization period for these patients, and to the best of our knowledge, no other study concerning the PLR value in snakebites has been conducted.

- We believe that the prediction of poor prognosis and the development of complication in snakebites are of vital importance, particularly in crowded hospitals with limited inpatient bed availability, including snakebite endemic regions, for patient monitoring and discharging.

\section{CONCLUSION}

We believe that it is possible to make predictions on hospitalization periods and development of complications using the NLR and PLR values, which are measured in patients while applying to ER for snakebites. Pre-treatment on admission NLR and PLR was significantly increased in the moderately and severely snake poisoned patients. Also, there was significant correlation of NLR with poorer outcomes ( ICU stay time and hospital stay time and complications).

It is recommended to use NLR and PLR as an early, easily, readily available, low cost and useful parameter to predict severity and direct therapy of snake poisoned patients specially in clinical settings lacking INR and other blood clotting tests. Further studies on larger scale to define more the prognostic role of NLR and PLR in snake poisoning are warranted

\section{REFERENCES}

1. Munjal Y. API textbook of medicine.

2. Gormeli Kurt N. The Importance of Neutrophil Lymphocyte Ratio (NLR) and Platelet Lymphocyte Ratio (PLR) for Prognosis and Hospitalization Period in Case of Snakebites. Eurasian Journal of Medical Investigation. 2018.

3. Bentur Y, Cahana A. Unusual local complications of Vipera palaestinae bite. Toxicon 2003;41:633-5.

4. Benvenuti LA, França FO, Barbaro KC, Nunes JR, Cardoso JL. Pulmonary haemorrhage causing rapid death after Bothrops jararacussu snakebite: a case report. Toxicon 2003;42:331-4.

5. Chippaux JP. Local complications of snake bites [Article in French]. Med Trop (Mars) 1982:42:177-83.

6. Firat C, Erbatur S, Aytekin AH, Klınç H. Effectiveness of early fasciotomy in the management of snakebites. Ulus Travma Acil Cerrahi Derg 2012;18:417-23.

7. Al B, Orak M, Aldemir M, Güloğlu C. Snakebites in adults from the Diyarbakir region in southeast Turkey. Ulus Travma Acil Cerrahi Derg 2010;16:210-4.

8. Kang, S.; Moon, J. and Chun B. (2016): Does the traditional snakebite severity score correctly classify envenomated patients? Clin Exp Emerg, 3(1): 34-40.

9. Khin, M.; Nyuntb, T.; Ooc, N. et al., (2012): Prognostic indicators in patients with snakebite: analysis of two-year data from a township hospital in central Myanmar. WHO South-East Asia Journal of Public Health, 1(2):144-150.

10. Isbister, G.: Maduwage, K.; Scorgie, F. et al., (2015): Venom concentrations and clotting factor levels in a prospective cohort of Russell's viper bites with coagulopathy. PLoS Negl Trop Dis, 9(8): 1-12.

11. Azab B, Bhatt VR, Phookan J, Murukutla S, Kohn N, Terjanian T, Usefulness of the neutrophil-to-lymphocyte ratio in predicting short- and long-term mortality in breast cancer patients. Ann Surg Oncol 2012;19:217-24.

12. Celikbilek A, Ismailogullari S, Zararsiz G. Neutrophil to lymphocyte ratio predicts poor prognosis in ischemic cerebrovascular disease. J Clin Lab Anal. 2014;28:27-31.

13. Gibson PH, Cuthbertson BH, Croal BL, Rae D, El-Shafei H, Gibson G, et al. Usefulness of neutrophil/lymphocyte ratio as predictor of new-onset atrial fibrillation after coronary artery bypass grafting. Am J Cardiol 2010;105:186-91.

14. Balta S, Ozturk C.The platelet-lymphocyte ratio: A simple, inexpensive and rapid prognostic marker for cardiovascular events. Platelets 2015;26:680-1.

15. Horne, B.; Anderson, J.; John, J. et al., (2005): Which white blood cell subtypes predict increased cardiovascular risk? J Am Coll Cardiol, 45:1638-1643.

16. de Jager, C.; Vanwijk, P; Mathoera, R. et al., (2010): Lymphocytopenia and neutrophil-lymphocyte count ratio predict bacteremia better than conventional infection markers in an emergency care unit. Crit Care, 14:R192.

17. Dundar, Z.; Ergin, M.; Koylu, R. et al., (2014): Neutrophil-lymphocyte ratio in patients with pesticide poisoning.The Journal of Emergency Medicine, 47(3):286-293

18. Moreira, V.; Gutie'rrez, J.; Amaral, R. et al., (2009): Effect of Bothrops asper snake venom on the expression of cyclooxygenases and production of prostaglandins by peritoneal leukocytes in vivo, and by isolated neutrophils and macrophages in vitro. Prostaglandins Leukot Essent Fatty Acids, 80: 107114.

19. Zornetta, I.; Caccin, P.; Fernandez, J. et al., (2012): Envenomations by Bothrops and Crotalus snakes induce the release of mitochondrial alarmins. PLoS Negl Trop Dis, 6(2): el526.

20. Elbey, B.; Baykal, B.; Yazgan, U. et al., (2015): The prognostic value of the 
neutrophil/ymphocyte ratio in patients with snake bites for clinical outcomes and complications. Saudi Journal of Biological Sciences.

21. Sharma, R.; Katkar, G.: Sundaram, M. et al., (2015): Oxidative stressinduced methemoglobinemia is the silent killer during snakebite: a nove and strategic neutralization by melatonin. J. Pineal Res, 59:240-254

22. Chacon, B.; Gutierrez, H.; Fallas, A. et al., (2015): Bacterial infections associated with Viperidae snakebites: a l4-year experience at the Hospital Nacional de Niños de Costa Rica.9th world congress of the world society for pediatric infectious diseases (WSPID) Rio De Janeiro Brazil November 18-20, 2015.

23. Acikalin, A. and Gokel, Y. (2011): Serum IL-6, TNFalpha levels in snakebite cases occurring in Southern Turkey. Emergency Med J, 28:208-21 1.

24. Stone, S. I Isbister G. Shahmy S et al., (2013): Immune response to snake envenoming and treatment with antivenom; complement activation, cytokine production and mast cell degranulation. PLoS Negl Trop Dis J, 7(7):e2326.

25. Al Durihim, H.; Al Hussaini, M.; Bin Salih, S. et al., (2010): Snake bite envenomation: experience at King Abdulaziz Medical City, Riyadh. East Mediterr Health J, 16(4): 438-441.

26. Isbister, G.; Gault, A.; Tasoulis, T. et al., (2016): A definite bite by the Ornamental Snake (Denisonia maculata) causing mild envenoming. Clin Toxicol (Phila), 54(3):241-244.

27. Akilli, N.; Yortanli, M.; Mutlu, H. et al., (2014): Prognostic importance of neutrophil-lymphocyte ratio in critically ill patients: short- and long-term outcomes. American Journal of Emergency Medicine, 32: 1476-1480.

28. Acanfora, D.; Gheorghiade, M.; Trojano, L. et al., (2001): Relative lymphocyte count: a prognostic indicator of mortality in elderly patients with congestive heart failure. Am Heart J, 142:167-173.

29. Kawahara T. Furuya, K. Nakamura, M. et al. (2016): Neutrophil-tolymphocyte ratio is a prognostic marker in bladder cancer patients after radical cystectomy. BMC Cancer, 16:185.

30. Kaya, M.; Akpek, M.; Lam, Y. et al., (2013): Prognostic value of neutrophil/lymphocyte ratio in patients with ST-elevated myocardial infarction undergoing primary coronary intervention: a prospective, multicenter study. Int J Cardiol, 168:1154-1159.

31. Salciccioli, J.; Marshall, D.; Pimentel, M. et al., (2015): The association between the neutrophil-to-lymphocyte ratio and mortality in critical illness: an observational cohort study. Crit Care, 19:13-18.

32. Balta, S.: Demirkol, S.: Hatipoglu, M. et al., (2013): Other inflammatory indicators should be kept in mind when assessing red cell distribution width in patients lymphocyte ratio can be a valuable marker in defining disease activity in patients who have started antitumor necrosis factor (TNF) drugs for ankylosing spondylitis. Eur J Rheumatol, 1: 101-105.

33. Zahorec, R. (2001): Ratio of neutrophil to lymphocyte counts: rapid and simple parameter of systemic inflammation and stress in critically ill. Bratisl LekListy, 102: 5-14

34. Alan $\alpha$, S.; Tuna, S. and Turkoglu, E. (2015): The relation of neutrophil-tolymphocyte ratio, platelet-to-lymphocyte ratio, and mean platelet volume with the presence and severity of Behcet's syndrome. Kaohsiung Journal of Medical Sciences, 31:626-631.

35. Okur Mi, Yildirnm AM, Kose R. Venomous Snake Bites And Its Therapy In Turkey. Turkiye Klinikleri J Med Sci 2001;21:528-32.

36. Koh DC, Armugam A, Jeyaseelan K. Snake venom components and their applications in biomedicine. Cell Mol Life Sci 2006;63:3030-41. 